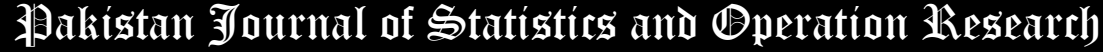

\section{Assessing the Lifetime Performance Index of Burr Type III Distribution under Progressive Type II Censoring}

\author{
Amal S. Hassan ${ }^{1}$, Amany S. Selmy², Salwa M. Assar ${ }^{*}$ \\ * Corresponding Author \\ 1. Department of Mathematical Statistics, Faculty of Graduate Studies for Statistical Research, Cairo University, \\ Egypt, dr.amalelmoslamy@gmail.com \\ 2. Department of Mathematical Statistics, Faculty of Graduate Studies for Statistical Research, Cairo University, \\ Egypt, amanyselmy34@ gmail.com \\ 3. Department of Mathematical Statistics, Faculty of Graduate Studies for Statistical Research, Cairo University, \\ Egypt, salwaassar@yahoo.com
}

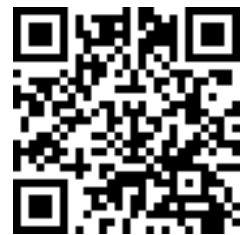

\begin{abstract}
Process capability analysis has been widely applied in the field of quality control to monitor the performance of industrial processes. Hence, lifetime performance index $\mathrm{C}_{\mathrm{L}}$ is used to measure the potential and performance of a process. In the present study, we construct a maximum likelihood estimator of $\mathrm{C}_{\mathrm{L}}$ under Burr type III distribution based on the progressive Type II censored sample. The maximum likelihood estimator of $\mathrm{C}_{\mathrm{L}}$ is then utilized to develop the hypothesis testing procedure in the condition of known L. Finally, one practical example and Monte Carlo simulation are given to assess the behavior of the lifetime performance index under given significance level.
\end{abstract}

Key Words: Process capability index; Lifetime performance index; Burr Type III distribution, Progressive Type II censoring; Maximum likelihood estimator

\section{Introduction}

Burr (1942) has suggested twelve types of cumulative distribution functions, which yield a variety of density shapes. The principal aim in choosing one of these forms of distributions is to facilitate the mathematical analysis to which it is subjected, while attaining a reasonable approximation. Burr Type III (BIII) distribution is a very important lifetime model in the analysis of equipment failure data, vehicle and informational studies, as well as in models of stress and durability. The lifetime $X$ of product has a two-parameter BIII distribution if its cumulative distribution function (cdf) and probability distribution function (pdf) defined respectively as

$$
F(x ; \lambda, \beta)=\left[1+\mathrm{x}^{-\lambda}\right]^{-\beta}, x>0, \lambda, \beta>0,
$$

and,

$$
f(x ; \lambda, \beta)=\lambda \beta x^{-\lambda-1}\left[1+x^{-\lambda}\right]^{-\beta-1} ; x>0 .
$$

where $\lambda$ and $\beta$ are shape parameters. Altindag et al. (2017) proposed the estimation and prediction problems for the BIII distribution under type II censored data. Panahi (2017) developed the statistical inference of the unknown parameters of a BIII distribution based on the unified hybrid censored sample. The maximum likelihood (ML) estimates of the unknown parameters were obtained using the expectation-maximization algorithm. Gamchi et al. (2019) studied the estimation and prediction problems for the BIII distribution under progressive type II hybrid censored data. They obtained the ML estimates of unknown parameters using stochastic expectation maximization algorithm.

Process capability indices (PCIs) have been used in the manufacturing industry to provide quantitative measures of process potential and performance. High quality production provides advantages such as cost saving, reduced scrap 
or remanufacturing, higher yield and increased customer satisfaction and market share. PCIs are extensively used in industry to evaluate the conformation of products (process yield) to their specifications. PCIs are utilized to assess whether product quality meets the required level. For manufacture industry PCI is an effective and convenient tool for quality assessment. There are several PCI in the literature that can be used to measure the capability of process. As a measure of quality for lifetime, Montgomery (1985) and Kane (1986) proposed the process capability index, $C_{L}$ for evaluating the lifetime performance of electronic components, where $\mathrm{L}$ is the lower specification limit.

In life-testing and reliability experiments, units may be lost or removed during experimentation before failure. The removal may be unplanned, like in accidental breakage of an experimental unit, or if a unit drops out of the experiment. Most often, the removal is pre-planned in order to save time and cost. Progressive censoring is an efficient in exploitation of the available resources. In other words, when some of the surviving units in the experiment are removed early, they can be used for some other tests. Cohen (1965) discussed the importance of progressive censoring in lifetesting reliability experiments. There are several types of censoring schemes in survival analysis, one of these schemes is the type II censoring scheme. A generalization of type II censoring is the progressive type II censoring (PTIIC) which allows for units to be removed from the test at points other than the final termination point.

Here, we consider a PTIIC scheme which is a useful scheme in which a specific fraction of individuals at risk may be removed from the experiment at each of several ordered failure times. Therefore, progressive censoring scheme allows us the removals (before the termination of the experiment) to incorporate into analysis.

Statistical inferences for $C_{L}$ based on censored samples have been considered by several researchers. For instance, Lee et al. (2009) proposed a hypothesis testing procedure based on the ML estimator of $C_{L}$ to evaluate the product quality for two-parameter exponential under PTIIC samples. Hong et al. (2012) applied the large-sample theory to construct a ML estimator of $C_{L}$ with the progressive first-failure-censored sampling plan under the Weibull distribution. The ML estimator of $C_{L}$ is then utilized to develop a new hypothesis testing procedure in the condition of known $L$. Ahmadi et al. (2013) constructed a ML estimator and lower bound of $C_{L}$ for Weibull distribution with a known shape parameter. The ML estimator was obtained on the basis of progressive first-failure censored data. Wu et al. (2013) constructed statistical methods of assessing the lifetime performance of products with Rayleigh distribution under PTIIC samples. The ML estimator of $C_{L}$ was inferred by the technique of data transformation. Wu et al. (2014) constructed the ML estimator of $C_{L}$ for the two-parameter Burr XII distribution with PTIIC sample on the condition of known $L$. Gildeh and Nadi (2016) obtained the uniformly minimum variance unbiased estimator (UMVUE) of $C_{L}$ under the assumption of two-parameter exponential distribution based on a progressive first-failure censored sample. Then the UMVUE of $C_{L}$ was utilized to develop the new hypothesis testing procedure. Hassanein (2018) devoted to evaluate $C_{L}$ for the three- parameter power Lomax distribution under progressive first-failure censoring samples with respect to a lower specification limit $L$. Wijekularathna and Subedi (2019) constructed the ML estimator of $C_{L}$ based on PTIIC sample. The ML estimator of $C_{L}$ was utilized to develop the hypothesis testing procedure.

Due to the importance of BIII distribution in practical framework and PTIIC scheme, so our objective here is to construct an ML estimator of $C_{L}$ for BIII distribution under PTIIC sample. The ML estimator of $C_{L}$ is then utilized to develop a new hypothesis testing procedure under the condition of a known lower specification limit $L$. The new testing procedure can be employed to assess whether the lifetime of products (or items) adheres to the required level in the condition of known $L$. The rest of this paper is organized as follows: Section 2 introduces some properties of the lifetime performance index for lifetime of product (or item) with the transformation of BIII distribution based on the PTIIC sample and discusses the relationship between the lifetime performance index and conforming rate. Section 3 presents the ML estimator of the $C_{L}$ and its statistical properties in view of theoretical and practical framework. Section 4 develops a new hypothesis testing procedure for lifetime performance index for PTIIC samples, the power function of test statistics is provided. Further, a simulation study of power function is conducted and confidence interval for $C_{L}$ is derived. Application to real data is discussed in Section 5. The article encloses with some concluding remarks.

\section{The Lifetime Performance Index and Conforming Rate}

Suppose that the lifetime $X$ of product has a two-parameter BIII distribution with the cdf and pdf defined in (1) and (2) respectively. To obtain $C_{L}$ for the BIII distribution, the following transformation will be used, 
Hence, the pdf of $Y$ is given by

$$
y=\log _{e}\left(1+x^{-\lambda}\right)
$$

$$
\begin{aligned}
& \qquad \begin{array}{c}
f(y ; \beta)=f(x ; \lambda, \beta)|\mathrm{J}|, \quad|\mathrm{J}|=|d x / d y|=\lambda^{-1}\left(1+x^{-\lambda}\right) x^{\lambda+1}, \\
f(y ; \beta)=\beta e^{-\beta y}, y>0, \beta>0, \\
\text { and } \quad F(y ; \beta)
\end{array} \quad \frac{d f(y ; \beta)}{d y}=\frac{d}{d y}\left(\beta e^{-\beta y}\right)=1-e^{-\beta y} .
\end{aligned}
$$

Also, it is known that the mean and variance of $Y$ are $1 / \beta$ and $1 / \beta^{2}$ respectively. The failure rate function $r(y ; \beta)$ is defined by

$$
r(y ; \beta)=\frac{f(y ; \beta)}{1-F(y ; \beta)}=\frac{\beta e^{-\beta y}}{e^{-\beta y}}=\beta .
$$

So, the lifetime performance index $C_{L}$ is obtained by substituting mean and variance as follows

$$
C_{L}=(\mu-L) / \sigma=1-\beta L,-\infty<C_{L}<1,
$$

where the process mean $\mu=1 / \beta$, the process standard deviation $\sigma=1 / \beta$, and $L$ is the lower specification limit. When the mean lifetime of products $1 / \beta>L$, then $C_{L}>0$. From (5) and (6), it can be seen that the larger value of the mean, the smaller value of failure rate and the lager value of $C_{L}$. Therefore, $C_{L}$ reasonably and accurately represents the lifetime performance of products.

If the lifetime of a product $Y$, which $Y \geq L$, and $\beta>0$ exceeds $L$, then the product is defined as a conforming product. The ratio of conforming products is known as the conforming rate $\left(P_{r}\right)$ and can be defined as

$$
P_{r}=P(Y \geq L)=\int_{L}^{\infty} f(y) d y=\int_{L}^{\infty} \beta e^{-\beta y} d y=e^{-\beta L} .
$$

From Equation (6), $\beta L=1-C_{L}$, then $P_{r}$ is as follows

$$
P_{r}=e^{\left(C_{L}-1\right)}, \quad-\infty<C_{L}<1 .
$$

Obviously, a strictly increasing relationship exists between $P_{r}$ and $C_{L}$. Thus, the larger value of $C_{L}$, the larger value of $P_{r}$. Table 1 lists various $C_{L}$ values and the corresponding $P_{r}$. For the $C_{L}$ values which are not listed in Table1, the $P_{r}$ can be obtained through interpolation. Utilizing the one-to-one relationship between $P_{r}$ and $C_{L}$, so the lifetime performance index can be a flexible and effective tool, not only for evaluating product quality, but also for estimating $P_{r}$.

Table 1: The lifetime performance index versus the conforming rate

\begin{tabular}{cccc}
\hline$Z$ & $P_{r}$ & $C_{L}$ & $P_{r}$ \\
\hline$-\infty$ & 0.00000 & 0.15 & 0.42741 \\
-9.00 & 0.00004 & 0.20 & 0.44933 \\
-8.00 & 0.00012 & 0.25 & 0.47237 \\
-7.00 & 0.00033 & 0.30 & 0.49659 \\
-6.00 & 0.00091 & 0.35 & 0.52205 \\
-5.00 & 0.00248 & 0.40 & 0.54881 \\
-4.50 & 0.00409 & 0.45 & 0.57695 \\
-4.00 & 0.00673 & 0.50 & 0.60653 \\
-3.50 & 0.01111 & 0.55 & 0.63763 \\
-3.00 & 0.01832 & 0.60 & 0.67032 \\
-2.50 & 0.03019 & 0.65 & 0.70469 \\
-2.00 & 0.04979 & 0.70 & 0.74082 \\
-1.50 & 0.08208 & 0.75 & 0.77880 \\
-1.00 & 0.13534 & 0.80 & 0.81873 \\
-0.50 & 0.22313 & 0.85 & 0.86071 \\
0.00 & 0.36788 & 0.90 & 0.90484 \\
0.05 & 0.38674 & 0.95 & 0.95123 \\
0.10 & 0.40657 & 1.00 & 1.00000 \\
\hline
\end{tabular}

\section{ML Estimator of Lifetime Performance Index}


In this section, ML of $C_{L}$ under BIII is obtained in presence of PTIIC sample. Based on transformation (3) we obtain the ML estimator of exponential distribution. Let $Y$ denote the lifetime $Y$ has a one-parameter exponential distribution with the pdf (4). The joint likelihood function of $y_{(1)}, y_{(2)}, \ldots, y_{(m)}$, based on PTII censoring is given as

$$
\begin{aligned}
L(\beta) & =C_{2} \prod_{i=1}^{m} f\left(y_{(i)}, \theta\right)\left[1-F\left(y_{(i)}\right)\right]^{R_{i}}=C_{2} \prod_{i=1}^{m}\left(\beta e^{-\beta y_{(i)}}\right)\left[1-\left(1-e^{-\beta y_{(i)}}\right)\right]^{R_{i}} \\
& =C_{2} \beta^{m} e^{-\beta \sum_{i=1}^{m}\left(1+R_{i}\right) y_{(i)}},
\end{aligned}
$$

where, $C_{2}=\left(n-R_{1}-1\right) \ldots\left(n-R_{1}-R_{2}-\ldots-R_{m-1}-m+1\right)$. The ML estimator $\hat{\beta}$ of $\beta$ can be obtained as

$$
\hat{\beta}=\frac{m}{\sum_{i=1}^{m}\left(1+R_{i}\right) y_{(i)}},
$$

where $R_{\mathrm{i}}$ 's denote the number of items removed at the time of the $i^{\text {th }}$ failure, $m$ be the number of failures observed before termination. By using the invariance property of ML estimator, hence the ML estimator of $C_{L}$, say $\hat{C}_{L}$ can be written as

$$
\hat{C}_{L}=1-\hat{\beta} L=1-\frac{m L}{\sum_{i=1}^{m}\left(1+R_{i}\right) y_{(i)}} .
$$

Let $W=\sum_{i=1}^{m}\left(1+R_{i}\right) y_{(i)}$, then the ML estimator $\hat{C}_{L}$ of $C_{L}$ can be rewritten as $\hat{C}_{L}=1-m L / W$. To prove that $2 \beta W \sim \chi_{(2 m)}^{2}$, the normalized spacing $D_{(i)}$ between $\mathrm{y}_{(1)}, \mathrm{y}_{(2)}, \ldots, \mathrm{y}_{(m)}$ is defined as follows

$$
D_{1}=n y_{(1)}, \quad D_{i}=\left[n-\sum_{j=1}^{i-1}\left(R_{j}+1\right)\right]\left(y_{(i)}-y_{(i-1)}\right), \quad i=1, \ldots, m
$$

Hence, $W=\sum_{i=1}^{m}\left(1+R_{i}\right) y_{(i)}=\sum_{i=1}^{m} D_{i}$.

It is known that the normalized spacing $D_{1}, D_{2}, \ldots, D_{m}$ constitute an independent random sample from oneparameter exponential distribution (see Viveros and Balakrishnan(1994)), hence

$$
2 \beta W=2 \beta \sum_{i=1}^{m} D_{i} \sim \chi_{(2 m)}^{2}
$$

Furthermore, the expectation of $\hat{C}_{L}$ can be derived as follows

$$
E\left(\hat{C}_{L}\right)=E\left(1-\frac{m L}{\sum_{i=1}^{m}\left(1+R_{i}\right) y_{i, n}}\right)=E\left(1-\frac{m L}{W}\right)=1-2 \beta m L E\left(\frac{1}{2 \beta W}\right),
$$

where $(1 / 2 \beta W)$ has the inverted chi-square distribution with mean $1 /(2 m-2)$. Here, the expected value of $\hat{C}_{L}$ is as follows

$$
E\left(\hat{C}_{L}\right)=1-\frac{\beta m L}{m-1},
$$

but $E\left(\hat{C}_{L}\right) \neq C_{L}$ where $C_{L}=1-\beta L$, the ML estimator $\hat{C}_{L}$ is not an unbiased estimator of $C_{L}$. But when $m \rightarrow \infty$, $E\left(\hat{C}_{L}\right) \rightarrow C_{L}$, so the ML estimator $\hat{C}_{L}$ is asymptotically unbiased estimator. Moreover, the mean square error (MSE) is defined as

$$
\operatorname{MSE}\left(\hat{C}_{L}\right)=E\left[\hat{C}_{L}-C_{L}\right]^{2}=\frac{\beta^{2} m^{2} L^{2}}{(m-1)^{2}(m-2)}+\frac{\beta^{2} L^{2}}{(m-1)^{2}} .
$$


In the following algorithm the numerical study is carried out to obtain the ML estimate of $C_{L}$ and their MSE for different sampling schemes. The simulation procedure is utilizing for 1000 random samples via $R$ 3.5.3. The following steps are created as follows

\section{Algorithm (1)}

Step 1: Generate a random sample $u_{1}, u_{2}, \ldots, u_{m}$ of size $m$ from a uniform $(0,1)$, then the uniform random numbers can be transformed to BIII random numbers by using inversion method

Step 2: The generated data for $y_{i}=\log _{e}\left(1+x_{i}^{-\lambda}\right), i=1, \ldots, m$, where $\left(x_{1}, x_{2}, \ldots, x_{m}\right)$ is a random sample from the BIII distribution.

Step 3: Set $\left(y_{(1)}, y_{(2)}, \ldots, y_{(m)}\right)$ is the PTC sample from a one-parameter exponential distribution with pdf (4).

Step 4: Given $\beta=(0.5,1$ and 1.5), $\lambda=1.7$ and $3.5, L=0.5$, the selected value of $(n, m)$ are $(n, m)=(10,5),(20,10)$, (30,5), (30,10), (50,5), (50,10) and $R=\left(R_{1}, R_{2}, \ldots, R_{m}\right)$ has different samples schemes. Three sample values are used for each case, for example $(n, m)=(10,5)$ the schemes are $R=(3,0,0,0,2),(1,1,1,1,1),(1,0,2,0,2)$.

Step 5: The value of $\hat{C}_{L}$ is calculated by using $\hat{C}_{L}=1-\left(m L / \sum_{i=1}^{m}\left(1+R_{i}\right) y_{(i)}\right)$.

Step 6: The MSE $\left(\hat{C}_{L}\right)=E\left[\hat{C}_{L}-C_{L}\right]^{2}$, where $C_{L}=1-\beta L$, is calculated. Numerical results are listed in Tables 2 and 3 .

Table 2: ML estimator and MSEs under PTIIC schemes for $\beta=0.5,1,1.5$ and $\lambda=1.7$

\begin{tabular}{|c|c|c|c|c|}
\hline$(n, m)$ & Scheme & $\beta$ & $\hat{C}_{L}$ & MSE \\
\hline \multirow{3}{*}{$(10,5)$} & \multirow{3}{*}{$(1,1,1,1,1)$} & 0.5 & 0.70447 & 0.09848 \\
\hline & & 1 & 0.58870 & 0.02994 \\
\hline & & 1.5 & 0.40663 & 0.03468 \\
\hline \multirow{3}{*}{$(20,5)$} & \multirow{3}{*}{$(3,3,3,3,3)$} & 0.5 & 0.69602 & 0.23974 \\
\hline & & 1 & 0.51405 & 0.05983 \\
\hline & & 1.5 & 0.40906 & 0.11195 \\
\hline \multirow{3}{*}{$(30,5)$} & \multirow{3}{*}{$(5,5,5,5,5)$} & 0.5 & 0.77243 & 0.01317 \\
\hline & & 1 & 0.51622 & 0.17160 \\
\hline & & 1.5 & 0.38998 & 0.07352 \\
\hline \multirow{3}{*}{$(50,5)$} & \multirow{3}{*}{$(9,9,9,9,9)$} & 0.5 & 0.75174 & 0.04122 \\
\hline & & 1 & 0.53072 & 0.03828 \\
\hline & & 1.5 & 0.46297 & 0.03530 \\
\hline
\end{tabular}

Table 3: ML estimator and MSEs under PTIIC schemes for $\beta=0.5,1,1.5$ and $\lambda=3.5$

\begin{tabular}{|c|c|c|c|c|}
\hline$(n, m)$ & Scheme & $\beta$ & $\hat{C}_{L}$ & MSE \\
\hline \multirow{3}{*}{$(10,5)$} & \multirow{3}{*}{$(1,1,1,1,1)$} & 0.5 & 0.93076 & 0.00049 \\
\hline & & 1 & 0.84412 & 0.00443 \\
\hline & & 1.5 & 0.78342 & 0.00555 \\
\hline \multirow{3}{*}{$(20,5)$} & \multirow{3}{*}{$(3,3,3,3,3)$} & 0.5 & 0.92954 & 0.00049 \\
\hline & & 1 & 0.84476 & 0.00259 \\
\hline & & 1.5 & 0.77620 & 0.00720 \\
\hline \multirow[b]{2}{*}{$(30,5)$} & \multirow[b]{2}{*}{$(5,5,5,5,5)$} & 0.5 & 0.92509 & 0.00106 \\
\hline & & 1 & 0.84812 & 0.00164 \\
\hline
\end{tabular}




\begin{tabular}{ccccc}
\hline$(n, m)$ & Scheme & $\beta$ & $\hat{C}_{L}$ & MSE \\
\hline \multirow{3}{*}{$(50,5)$} & & 1.5 & 0.78081 & 0.00719 \\
& & 0.5 & 0.92190 & 0.00114 \\
\cline { 3 - 5 } & $(9,9,9,9,9)$ & 1 & 0.84351 & 0.00500 \\
\cline { 3 - 5 } & & 1.5 & 0.77793 & 0.00526 \\
\hline
\end{tabular}

From these tables, the following observations can be detected about the performance of ML estimate $C_{L}$,

$>$ The MSEs of the $\hat{C}_{L}$ estimates decrease as the sample sizes increase for different selected set of parameters for different samples schemes.

$>$ At $\lambda=1.7$ and as the value of $\beta$ increases, the ML estimates of $C_{L}$ decrease.

$>$ At $\beta=1$, the values of $\hat{C}_{L}$ are increasing as value of $\lambda$ increases for different values of $(n, m)$.

$>$ All results of Tables 2 and 3 show that the MSE are enough small and the scope of MSE is between 0.000 and 0.240 .

$>$ The estimated values(5,5,5,5,5), $R=$ and MSE varies depending on the values schemes for example $\hat{C}_{L}$ $(10,0,5,0,10),(0,0,5,0,20)$ for $(n, m)=(30,5)$. The estimated values $(0.77243,0.70431,0.64693)$ and $=\hat{C}_{L}$ MSE $=(0.0131,0.04056,0.1128)$ respectively at $\lambda=1.7, \beta=0.5$.

$>$ Hence, these results from the simulation studies illustrate that the performance of our proposed method is acceptable.

\section{Testing Procedure for the Lifetime Performance Index}

In this section, a statistical testing procedure is considered to assess whether the lifetime performance index adheres to the required level. The one-sided hypothesis testing and the power of test are discussed under PTII censoring. Assuming that the required index value of lifetime performance index is larger than $c^{*}$, where $c^{*}$ denotes the target value is constructed. The aim is to test the null hypothesis

$$
H_{0}: C_{L} \leq c^{*} \text { (the product is unreliable), }
$$

the alternative hypothesis

$$
H_{1}: C_{L}>c^{*} \text { (the product is reliable), }
$$

are constructed. The ML estimator $\hat{C}_{L}$ of $C_{L}$ is used as the test statistic, so the rejection region can be expressed as $\left\{\hat{C}_{L} \mid C_{L}>C_{0}^{*}\right\}$. Given the specified significance level $\gamma$, the critical value $C_{0}^{*}$ can be calculated as follows

$$
\begin{gathered}
P\left(\hat{C}_{L}>C_{0}^{*} \mid C_{L} \leq c^{*}\right) \leq \gamma \rightarrow P\left(1-\frac{m L}{W}>C_{0}^{*} \mid C_{L} \leq c^{*}\right) \leq \gamma \\
P\left(1-\frac{2 \beta m L}{2 \beta W}>C_{0}^{*} \mid C_{L} \leq c^{*}\right) \leq \gamma \rightarrow \sup _{C_{L} \leq c^{*}} P\left(2 \beta W>\frac{2 m\left(1-C_{L}\right)}{1-C_{0}^{*}}\right)=\gamma \\
P\left(2 \beta W \leq \frac{2 m\left(1-c^{*}\right)}{1-C_{0}^{*}}\right)=1-\gamma,
\end{gathered}
$$

$\left(\right.$ Since $P\left(2 \beta W>\frac{2 m\left(1-c^{*}\right)}{1-C_{0}^{*}}\right)$ as $\left.C_{L} \leq c^{*}\right)$ where $2 \beta W \sim \chi_{(2 m)}^{2}$. From (14), utilizing inverse-chi-square (INVCHI) $(1-\gamma, 2 m)$ function which represents the lower $1-\gamma$ percentile of $\chi_{(2 m)}^{2}$, then $\frac{2 m\left(1-c^{*}\right)}{1-C_{0}^{*}}=\operatorname{INVCHI}(1-\gamma, 2 m)$ is obtained. Thus, the critical value can be derived as; 


$$
C_{0}^{*}=1-\frac{2 m\left(1-c^{*}\right)}{\operatorname{INVCHI}(1-\gamma, 2 \mathrm{~m})},
$$

where $c^{*}, \gamma$ and $m$ denote the target value, the specified significance level and the number of observed failures before termination, respectively. Moreover, it can be found that $C_{0}^{*}$ is independent of $n$ and $R_{i}, \mathrm{i}=1,2, \ldots, m$.

\subsection{Power Function of the Test}

The power of this statistical test is the probability of correctly rejecting a false null hypothesis. The null hypothesis $H_{0}: C_{L} \leq c^{*}$, and the alternative hypothesis $H_{1}: C_{L}>c^{*}$, are constructed. The power of this statistical test is derived as follows:

Under PTIIC scheme, we get a size $\gamma$ test with the rejection region $\left\{\hat{C}_{L} \mid \hat{C}_{L}>1-\frac{2 m\left(1-c^{*}\right)}{\operatorname{INVCHI}(1-\gamma, 2 m)}\right\}$, for the number of observed failures before termination $\mathrm{m}$ and sample size $n(m \leq n)$. The power $P\left(C_{L}\right)$ of the test at this point $C_{L}>$ $c^{*}$ defined as;

$$
\begin{aligned}
P\left(C_{L}\right) & =p\left(\hat{C}_{L}>1-\frac{2 m\left(1-c^{*}\right)}{\operatorname{INVCHI}(1-\gamma, 2 m)}\right)=p\left(1-\frac{m L}{W}>1-\frac{2 m\left(1-c^{*}\right)}{\operatorname{INVCHI}(1-\gamma, 2 m)} \mid \beta=\frac{1-C_{L}}{L}\right) \\
& =p\left(2 \beta W>\frac{\beta L \operatorname{INVCHI}(1-\gamma, 2 m)}{\left(1-c^{*}\right)}\right)=p\left(2 \beta W>\frac{\left(1-C_{L}\right) \operatorname{INVCHI}(1-\gamma, 2 m)}{\left(1-c^{*}\right)}\right),
\end{aligned}
$$

where $2 \beta W \sim \chi_{(2 m)}^{2}$.

\section{Algorithm (2)}

The numerical study is designed to obtain the critical values of the power test. The simulated procedures are done via R 3.5.3 and described as follows

\section{Step 1:}

(a) Generation of data $\left(y_{1}, y_{2}, \ldots, y_{m}\right)$ is done as give in Algorithm 1.

(b) Set $\left(y_{(1)}, y_{(2)}, \ldots, y_{(m)}\right)$ as the PTIIC from a one-parameter exponential distribution with pdf (4).

(c) Given $c^{*}=0.1, C_{L}=0.1,0.2,0.3 \ldots 0.9, \lambda=1.7$ and 3.5, $L=0.5$, the indicated significance levels of $\gamma=0.01$ and 0.05 . The selected values of $(n, m)$ are $(n, m)=(10,5),(20,5),(30,5),(50,5)$, where $c^{*}<C_{L}<1$ and $m<n$ and $R=\left(R_{l}, R_{2}, \ldots R_{m}\right)$ different samples schemes

(d)The value of $\hat{C}_{L}$ is calculated by $\hat{C}_{L}=1-\frac{m L}{\sum_{i=1}^{m}\left(1+R_{i}\right) y_{(i)}}$.

(e) If $\hat{C}_{L}>C_{0}^{*}$, the critical value is obtained by using

Step2: (a) The Step1 is repeated1000 times.

$$
C_{0}^{*}=1-\frac{2 m\left(1-c^{*}\right)}{\operatorname{INVCHI}(1-\gamma, 2 m)} \text {. }
$$

(b)The estimation of the power $P\left(C_{L}\right)$, is $\hat{P}\left(C_{L}\right)=\frac{\text { Total Count }}{1000}$.

Step3: (a) The Step 2, based on 100 estimations of the power $P\left(C_{L}\right)$, can be obtained as follows: $\hat{P}_{1}\left(C_{L}\right), \hat{P}_{2}\left(C_{L}\right), \ldots . ., \hat{P}_{100}\left(C_{L}\right)$.

(b)The mean $\overline{\hat{P}\left(C_{L}\right)}$ of $\hat{P}_{1}\left(C_{L}\right), \hat{P}_{2}\left(C_{L}\right), \ldots . ., \hat{P}_{100}\left(C_{L}\right)$, that is 


$$
\overline{\hat{P}\left(C_{L}\right)}=\frac{\sum_{i=1}^{100} \hat{P}_{i}\left(C_{L}\right)}{100}, \text { is calculated. }
$$

(c)The MSE of $\hat{P}_{1}\left(C_{L}\right), \hat{P}_{2}\left(C_{L}\right), \ldots . ., \hat{P}_{100}\left(C_{L}\right)$ is obtained as follows

$$
\text { MSE }=\frac{\sum_{i=1}^{100}\left(\hat{P}_{i}\left(C_{L}\right)-P\left(C_{L}\right)\right)^{2}}{100}, \text { where } P\left(C_{L}\right) \text {, can be calculated from (16). }
$$

The results of simulated data are listed in Tables 4 to 7 .

Table 4: The values of $P\left(C_{L}\right), \overline{\hat{P}\left(C_{L}\right)}$ and MSE for test of hypothesis when $\lambda=\mathbf{1 . 7 ,} \boldsymbol{m = 5}$ and $\gamma=0.01$ under

\begin{tabular}{|c|c|c|c|c|c|}
\hline $\operatorname{Scheme}(R)$ & $\bar{n}$ & $\overline{C_{L}}$ & $P\left(C_{L}\right)$ & $\overline{\overline{\hat{P}\left(C_{L}\right)}}$ & $\overline{M S E}$ \\
\hline \multirow{9}{*}{$(1,1,1,1,1)$} & \multirow{9}{*}{10} & 0.1 & 0.058 & 0.07654 & 0.00007 \\
\hline & & 0.2 & 0.108 & 0.13321 & 0.00012 \\
\hline & & 0.3 & 0.212 & 0.21836 & 0.00014 \\
\hline & & 0.4 & 0.341 & 0.33785 & 0.00016 \\
\hline & & 0.5 & 0.488 & 0.48727 & 0.00025 \\
\hline & & 0.6 & 0.641 & 0.65523 & 0.00021 \\
\hline & & 0.7 & 0.804 & 0.80467 & 0.00014 \\
\hline & & 0.8 & 0.918 & 0.91568 & 0.00006 \\
\hline & & 0.9 & 0.982 & 0.9762 & 0.00002 \\
\hline \multirow{9}{*}{$(3,3,3,3,3)$} & \multirow{9}{*}{20} & 0.1 & 0.091 & 0.07635 & 0.00007 \\
\hline & & 0.2 & 0.152 & 0.13279 & 0.00010 \\
\hline & & 0.3 & 0.238 & 0.2152 & 0.00016 \\
\hline & & 0.4 & 0.341 & 0.33446 & 0.00020 \\
\hline & & 0.5 & 0.473 & 0.48963 & 0.00030 \\
\hline & & 0.6 & 0.68 & 0.65652 & 0.00024 \\
\hline & & 0.7 & 0.781 & 0.80792 & 0.00013 \\
\hline & & 0.8 & 0.919 & 0.91628 & 0.00008 \\
\hline & & 0.9 & 0.985 & 0.97724 & 0.00001 \\
\hline \multirow{9}{*}{$(5,5,5,5,5)$} & \multirow{9}{*}{30} & 0.1 & 0.077 & 0.07847 & 0.00008 \\
\hline & & 0.2 & 0.122 & 0.13236 & 0.00011 \\
\hline & & 0.3 & 0.22 & 0.21631 & 0.00017 \\
\hline & & 0.4 & 0.347 & 0.33632 & 0.00022 \\
\hline & & 0.5 & 0.506 & 0.48863 & 0.00016 \\
\hline & & 0.6 & 0.674 & 0.65341 & 0.00020 \\
\hline & & 0.7 & 0.814 & 0.808 & 0.00014 \\
\hline & & 0.8 & 0.905 & 0.91751 & 0.00007 \\
\hline & & 0.9 & 0.975 & 0.97696 & 0.00002 \\
\hline \multirow{3}{*}{$(9,9,9,9,9)$} & & 0.1 & 0.089 & 0.07711 & 0.00008 \\
\hline & & 0.2 & 0.153 & 0.1315 & 0.00009 \\
\hline & & 0.3 & 0.209 & 0.21716 & 0.00015 \\
\hline
\end{tabular}
PTIIC schemes 


\begin{tabular}{lccccc}
\hline Scheme(R) & $\boldsymbol{n}$ & $C_{L}$ & $P\left(C_{L}\right)$ & $\overline{\hat{P}\left(C_{L}\right)}$ & MSE \\
\hline \multirow{4}{*}{50} & & 0.4 & 0.348 & 0.33829 & 0.00023 \\
\cline { 2 - 5 } & & 0.5 & 0.492 & 0.49053 & 0.00026 \\
\cline { 2 - 5 } & & 0.6 & 0.655 & 0.65403 & 0.00028 \\
\cline { 2 - 5 } & & 0.7 & 0.79 & 0.80662 & 0.00019 \\
\cline { 2 - 5 } & & 0.8 & 0.915 & 0.91729 & 0.00009 \\
\cline { 2 - 5 } & & 0.9 & 0.98 & 0.97674 & 0.00002 \\
\hline
\end{tabular}

Table 5: The values of $P\left(C_{L}\right), \overline{\hat{P}\left(C_{L}\right)}$ and MSE for test of hypothesis when $\lambda=\mathbf{3 . 5}, \mathbf{m}=\mathbf{5}$ and $\gamma=0.01$ under PTIIC schemes

\begin{tabular}{|c|c|c|c|c|c|}
\hline $\operatorname{Scheme}(R)$ & $n$ & $C_{L}$ & $P\left(C_{L}\right)$ & $\overline{\hat{\hat{P}\left(C_{L}\right)}}$ & MSE \\
\hline \multirow{9}{*}{$(1,1,1,1,1)$} & \multirow{9}{*}{10} & 0.1 & 0.915 & 0.90585 & 0.00008 \\
\hline & & 0.2 & 0.951 & 0.9444 & 0.00005 \\
\hline & & 0.3 & 0.959 & 0.96884 & 0.00003 \\
\hline & & 0.4 & 0.979 & 0.98421 & 0.00001 \\
\hline & & 0.5 & 0.994 & 0.99249 & 0.00000 \\
\hline & & 0.6 & 0.996 & 0.99709 & 0.00000 \\
\hline & & 0.7 & 0.999 & 0.99911 & 0.00000 \\
\hline & & 0.8 & 0.998 & 0.99965 & 0.00000 \\
\hline & & 0.9 & 1 & 0.99997 & 0.00000 \\
\hline \multirow{9}{*}{$(3,3,3,3,3)$} & \multirow{9}{*}{20} & 0.1 & 0.89 & 0.90656 & 0.00005 \\
\hline & & 0.2 & 0.944 & 0.94484 & 0.00004 \\
\hline & & 0.3 & 0.979 & 0.96909 & 0.00003 \\
\hline & & 0.4 & 0.991 & 0.98398 & 0.00001 \\
\hline & & 0.5 & 0.993 & 0.99282 & 0.00000 \\
\hline & & 0.6 & 0.996 & 0.99701 & 0.00000 \\
\hline & & 0.7 & 1 & 0.99898 & 0.00000 \\
\hline & & 0.8 & 1 & 0.99973 & 0.00000 \\
\hline & & 0.9 & 0.999 & 0.99993 & 0.00000 \\
\hline \multirow{9}{*}{$(5,5,5,5,5)$} & \multirow{9}{*}{30} & 0.1 & 0.917 & 0.90679 & 0.00008 \\
\hline & & 0.2 & 0.949 & 0.94386 & 0.00004 \\
\hline & & 0.3 & 0.976 & 0.96847 & 0.00003 \\
\hline & & 0.4 & 0.981 & 0.98356 & 0.00001 \\
\hline & & 0.5 & 0.995 & 0.99311 & 0.00000 \\
\hline & & 0.6 & 0.995 & 0.99707 & 0.00000 \\
\hline & & 0.7 & 0.998 & 0.99901 & 0.00000 \\
\hline & & 0.8 & 0.999 & 0.99975 & 0.00000 \\
\hline & & 0.9 & 1 & 0.99992 & 0.00000 \\
\hline \multirow{3}{*}{$(9,9,9,9,9)$} & & 0.1 & 0.909 & 0.90746 & 0.00007 \\
\hline & & 0.2 & 0.943 & 0.94317 & 0.00005 \\
\hline & & 0.3 & 0.971 & 0.96884 & 0.00003 \\
\hline
\end{tabular}




\begin{tabular}{lccccc}
\hline Scheme $(\boldsymbol{R})$ & $\boldsymbol{n}$ & $C_{L}$ & $P\left(C_{L}\right)$ & $\overline{\hat{P}\left(C_{L}\right)}$ & MSE \\
\hline \multirow{3}{*}{50} & & 0.4 & 0.982 & 0.98419 & 0.00001 \\
\cline { 2 - 5 } & & 0.5 & 0.995 & 0.99223 & 0.00000 \\
\cline { 2 - 5 } & 0.6 & 0.998 & 0.99732 & 0.00000 \\
\cline { 2 - 5 } & & 0.7 & 0.999 & 0.99915 & 0.00000 \\
\cline { 2 - 5 } & & 0.8 & 1 & 0.99979 & 0.00000 \\
\cline { 2 - 5 } & & 0.9 & 1 & 0.99998 & 0.00000 \\
\hline
\end{tabular}

Table 6: The values of $P\left(C_{L}\right), \overline{\hat{P}\left(C_{L}\right)}$ and MSE for test of hypothesis when $\lambda=1.7, \mathbf{m}=5$ and $\gamma=0.05$ under PTIIC schemes

\begin{tabular}{|c|c|c|c|c|c|}
\hline $\operatorname{Scheme}(R)$ & $n$ & $C_{L}$ & $P\left(C_{L}\right)$ & $\overline{\hat{\hat{P}\left(C_{L}\right)}}$ & MSE \\
\hline \multirow{9}{*}{$(1,1,1,1,1)$} & \multirow{9}{*}{10} & 0.1 & 0.215 & 0.23066 & 0.00019 \\
\hline & & 0.2 & 0.306 & 0.32235 & 0.00016 \\
\hline & & 0.3 & 0.435 & 0.43078 & 0.00022 \\
\hline & & 0.4 & 0.563 & 0.55682 & 0.00026 \\
\hline & & 0.5 & 0.656 & 0.68056 & 0.00024 \\
\hline & & 0.6 & 0.788 & 0.79711 & 0.00015 \\
\hline & & 0.7 & 0.889 & 0.88896 & 0.00010 \\
\hline & & 0.8 & 0.945 & 0.94949 & 0.00003 \\
\hline & & 0.9 & 0.99 & 0.98341 & 0.00001 \\
\hline \multirow{9}{*}{$(3,3,3,3,3)$} & \multirow{9}{*}{20} & 0.1 & 0.242 & 0.23456 & 0.00014 \\
\hline & & 0.2 & 0.306 & 0.32361 & 0.00014 \\
\hline & & 0.3 & 0.426 & 0.43249 & 0.00024 \\
\hline & & 0.4 & 0.561 & 0.55938 & 0.00023 \\
\hline & & 0.5 & 0.688 & 0.68303 & 0.00021 \\
\hline & & 0.6 & 0.798 & 0.79969 & 0.00015 \\
\hline & & 0.7 & 0.893 & 0.88776 & 0.00009 \\
\hline & & 0.8 & 0.948 & 0.95141 & 0.00003 \\
\hline & & 0.9 & 0.983 & 0.98352 & 0.00001 \\
\hline \multirow{9}{*}{$(5,5,5,5,5)$} & \multirow{9}{*}{30} & 0.1 & 0.218 & 0.23352 & 0.00013 \\
\hline & & 0.2 & 0.313 & 0.32305 & 0.00022 \\
\hline & & 0.3 & 0.437 & 0.43378 & 0.00026 \\
\hline & & 0.4 & 0.573 & 0.55671 & 0.00027 \\
\hline & & 0.5 & 0.676 & 0.68274 & 0.00026 \\
\hline & & 0.6 & 0.802 & 0.79774 & 0.00014 \\
\hline & & 0.7 & 0.888 & 0.88973 & 0.00008 \\
\hline & & 0.8 & 0.952 & 0.94994 & 0.00004 \\
\hline & & 0.9 & 0.978 & 0.98422 & 0.00001 \\
\hline \multirow{6}{*}{$(9,9,9,9,9)$} & \multirow{6}{*}{50} & 0.1 & 0.201 & 0.23435 & 0.00017 \\
\hline & & 0.2 & 0.342 & 0.32295 & 0.00023 \\
\hline & & 0.3 & 0.415 & 0.43544 & 0.00025 \\
\hline & & 0.4 & 0.552 & 0.55546 & 0.00024 \\
\hline & & 0.5 & 0.679 & 0.68289 & 0.00020 \\
\hline & & 0.6 & 0.797 & 0.79875 & 0.00012 \\
\hline
\end{tabular}




\begin{tabular}{cccccc}
\hline Scheme $(\boldsymbol{R})$ & $\boldsymbol{n}$ & $C_{L}$ & $P\left(C_{L}\right)$ & $\overline{\hat{P}\left(C_{L}\right)}$ & MSE \\
\hline & & 0.7 & 0.897 & 0.89056 & 0.00008 \\
\cline { 2 - 5 } & 0.8 & 0.953 & 0.95005 & 0.00003 \\
\cline { 2 - 5 } & 0.9 & 0.988 & 0.98402 & 0.00001 \\
\hline
\end{tabular}

Table 7: The values of $P\left(C_{L}\right), \overline{\hat{P}\left(C_{L}\right)}$ and MSE for test of hypothesis when $\lambda=3.5, \boldsymbol{m = 5}$ and $\gamma=0.05$ under PTIIC schemes

\begin{tabular}{|c|c|c|c|c|c|}
\hline $\operatorname{Scheme}(R)$ & $n$ & $C_{L}$ & $P\left(C_{L}\right)$ & $\overline{\hat{P}\left(C_{L}\right)}$ & MSE \\
\hline \multirow{9}{*}{$(1,1,1,1,1)$} & \multirow{9}{*}{10} & 0.1 & 0.974 & 0.97088 & 0.00002 \\
\hline & & 0.2 & 0.985 & 0.98354 & 0.00001 \\
\hline & & 0.3 & 0.992 & 0.99036 & 0.00000 \\
\hline & & 0.4 & 0.994 & 0.995 & 0.00000 \\
\hline & & 0.5 & 0.997 & 0.99749 & 0.00000 \\
\hline & & 0.6 & 1 & 0.99906 & 0.00000 \\
\hline & & 0.7 & 0.999 & 0.99962 & 0.00000 \\
\hline & & 0.8 & 1 & 0.99986 & 0.00000 \\
\hline & & 0.9 & 1 & 0.99997 & 0.00000 \\
\hline \multirow{9}{*}{$(3,3,3,3,3)$} & \multirow{9}{*}{20} & 0.1 & 0.973 & 0.97135 & 0.00002 \\
\hline & & 0.2 & 0.983 & 0.98202 & 0.00001 \\
\hline & & 0.3 & 0.989 & 0.99014 & 0.00000 \\
\hline & & 0.4 & 0.996 & 0.99506 & 0.00000 \\
\hline & & 0.5 & 0.997 & 0.99783 & 0.00000 \\
\hline & & 0.6 & 0.998 & 0.99896 & 0.00000 \\
\hline & & 0.7 & 1 & 0.99957 & 0.00000 \\
\hline & & 0.8 & 1 & 0.99988 & 0.00000 \\
\hline & & 0.9 & 1 & 0.99995 & 0.00000 \\
\hline \multirow{9}{*}{$(5,5,5,5,5)$} & \multirow{9}{*}{30} & 0.1 & 0.96 & 0.97049 & 0.00003 \\
\hline & & 0.2 & 0.991 & 0.983 & 0.00001 \\
\hline & & 0.3 & 0.991 & 0.99018 & 0.00000 \\
\hline & & 0.4 & 0.999 & 0.99517 & 0.00000 \\
\hline & & 0.5 & 0.997 & 0.99729 & 0.00000 \\
\hline & & 0.6 & 0.999 & 0.99905 & 0.00000 \\
\hline & & 0.7 & 0.998 & 0.99956 & 0.00000 \\
\hline & & 0.8 & 1 & 0.99991 & 0.00000 \\
\hline & & 0.9 & 1 & 0.99995 & 0.00000 \\
\hline \multirow{8}{*}{$(9,9,9,9,9)$} & \multirow{8}{*}{50} & 0.1 & 0.967 & 0.97158 & 0.00002 \\
\hline & & 0.2 & 0.987 & 0.98254 & 0.00001 \\
\hline & & 0.3 & 0.991 & 0.99025 & 0.00001 \\
\hline & & 0.4 & 0.996 & 0.9948 & 0.00000 \\
\hline & & 0.5 & 0.994 & 0.99749 & 0.00000 \\
\hline & & 0.6 & 1 & 0.99899 & 0.00000 \\
\hline & & 0.7 & 1 & 0.99957 & 0.00000 \\
\hline & & 0.8 & 1 & 0.99989 & 0.00000 \\
\hline
\end{tabular}




\begin{tabular}{cccccc}
\hline $\operatorname{Scheme}(\boldsymbol{R})$ & $n$ & $C_{L}$ & $P\left(C_{L}\right)$ & $\overline{\hat{P}\left(C_{L}\right)}$ & MSE \\
\hline & & 0.9 & 1 & 1 & 0.00000 \\
\hline
\end{tabular}

From Tables 4 to 7 , the following observations can be detected as follows

$>$ For fixed $C_{L}$, as the observed number before termination $m$ increases the simulation power $\overline{\hat{P}\left(C_{L}\right)}$ and the power $P\left(C_{L}\right)$, increase (see for example Figure 1).

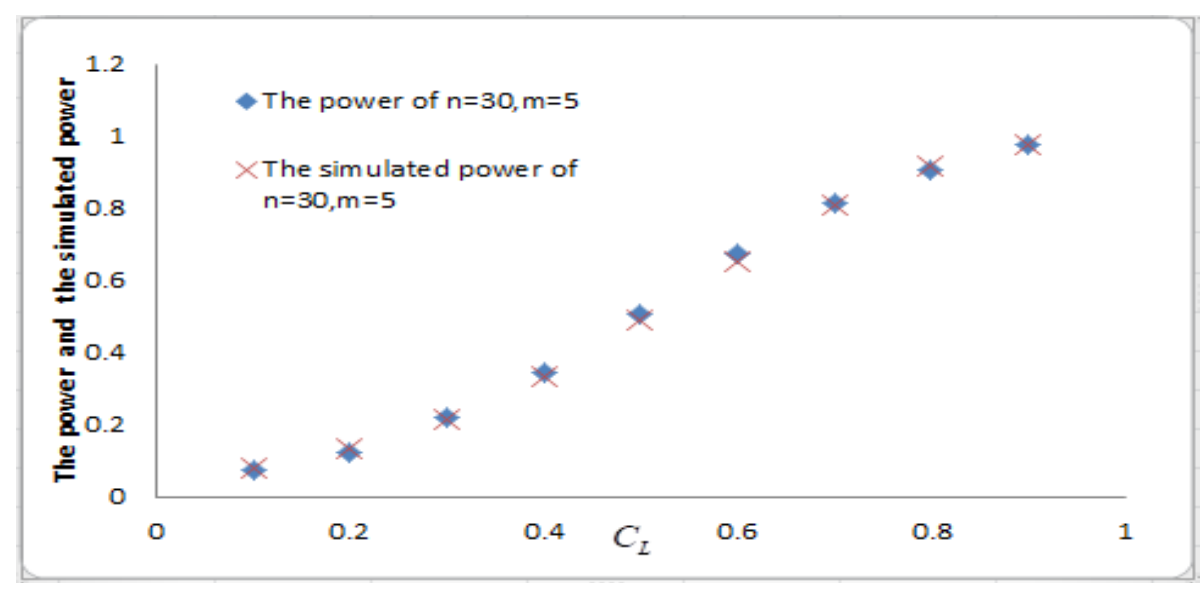

Figure 1: The power function of test at $\gamma=0.01, \lambda=1.7$ under PTIIC scheme for, $n=30, m=5$

$>$ For fixed $m$, the simulation power $\overline{\hat{P}\left(C_{L}\right)}$ and the power $P\left(C_{L}\right)$, increase when $C_{L}$ is increasing, but independent of $n$ and $R=\left(R_{1}, R_{2}, \ldots, R_{m}\right)$.

$>$ All of the simulation power $\overline{\hat{P}\left(C_{L}\right)}$ close to the power $P\left(C_{L}\right)$, for all value of $C_{L}$.

$>$ Values of the MSE are enough small and the scope of MSE is between 0.00000 and 0.00030 .

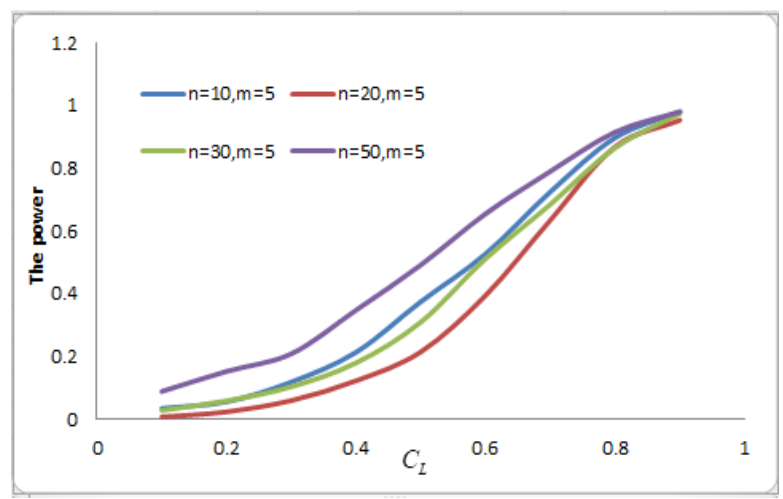

Figure 2: The power function of test at $\lambda=1.7$, $\gamma=0.01$ under different PTIIC schemes

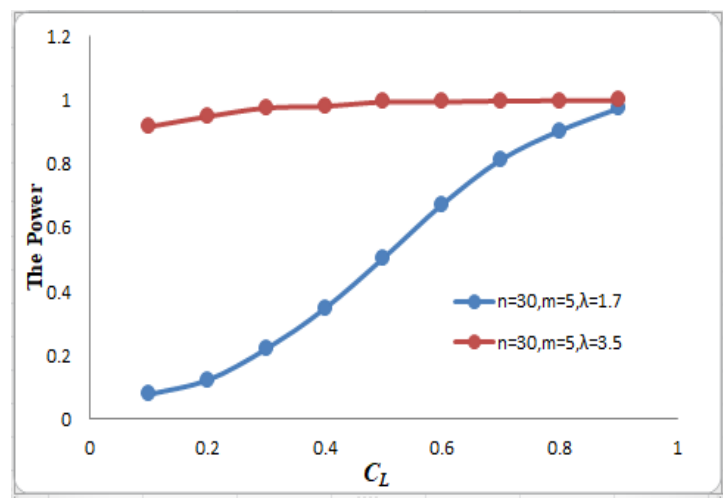

Figure 3: The power function of test at $\lambda=1.7$, and 3.5, $\gamma=0.01$ under PTIIC scheme for $n=30$, $m=5$

$>$ The power values, $P\left(C_{L}\right)$, increase when $C_{L}$ increases for different values of $n$ and at $m=5$ (see for example Figure 2).

$>$ The values of power function of test increase as the value of $\lambda$ increases (see for example Figure 3 ). 
Hence, these results from the simulation studies illustrate that the performance of our proposed method is acceptable

\subsection{Confidence Interval for $C_{L}$}

In this section, given the specified significance level $\gamma$, the level $(1-\gamma)$ one-sided confidence interval (CI) for $C_{L}$ can be derived as follows:

Since the pivotal quantity $2 \beta W$, where $2 \beta W \sim \chi^{2}{ }_{(2 m)}$ and INVCHI $(1-\gamma, 2 \mathrm{~m})$ which represents the lower $1-\gamma$ percentile of $\chi^{2}{ }_{(2 m)}$. So

$$
P(2 \beta W \leq \operatorname{INVCHI}(1-\gamma, 2 m))=1-\gamma,
$$

where $C_{L}=1-\beta L, C_{L}=1-\beta L$ and $\hat{C}_{L}=1-m L / W$, multiply in Equality (17) by $\hat{\beta} / 2 m$, then

$$
P\left(\frac{2 \beta W \hat{\beta} L}{2 m} \leq \frac{\hat{\beta} L \operatorname{INVCHI}(1-\gamma, 2 \mathrm{~m})}{2 m}\right)=1-\gamma,
$$

But, $\hat{\beta}=\frac{m}{W}$, then

$$
P\left(C_{L} \geq 1-\frac{\left(1-\hat{C}_{L}\right) \operatorname{INVCHI}(1-\gamma, 2 \mathrm{~m})}{2 m}\right)=1-\gamma .
$$

From Equation (19), then

$$
C_{L} \geq 1-\frac{\left(1-\hat{C}_{L}\right) \operatorname{INVCHI}(1-\gamma, 2 \mathrm{~m})}{2 m} .
$$

The level $(1-\gamma)$ one-sided is CI for $C_{L}$. Thus, the level $(1-\gamma)$ lower confidence bound $(L B)$ for $C_{L}$ can be written as:

$$
L B=1-\frac{\left(1-\hat{C}_{L}\right) \operatorname{INVCHI}(1-\gamma, 2 \mathrm{~m})}{2 m},
$$

where $\hat{C}_{L}, \gamma$ and $m$ denote the ML estimator of $C_{L}$, the specified significance level and the number of observed failures before termination, respectively.

\section{Application to Real Data}

A real data set was given by Mann and Fertig (1973) and recently discussed by Altindag et al. (2017). The data set contains 10 failure times of airplane components of total 13 items. The observed failure times are given in Table 8 .

Table 8: The observed failure times of censoring scheme

\begin{tabular}{|c|c|c|c|c|c|}
\hline$i$ & 1 & 2 & 3 & 4 & 5 \\
\hline$x_{i}$ & 0.22 & 0.50 & 0.88 & 1.00 & 1.32 \\
\hline$i$ & 6 & 7 & 8 & 9 & 10 \\
\hline$x_{i}$ & 1.33 & 1.54 & 1.76 & 2.50 & 3.00 \\
\hline
\end{tabular}

Then by using the transformed PTIIC samples with transformation $y=\log _{e}\left(1+x^{-\lambda}\right)$ is $y_{(i)}=(0.18,0.23,0.36,0.42$, $0.50,0.51,0.69,0.79,1.34,2.37)$, and let $R_{i}=(0,1,0,0,1,0,0,1,0,0), \gamma=0.05, L=0.1$. The performance index value set at $c^{*}=0.80$. The testing hypothesis $H_{0}: C_{L} \leq c^{*}$, versus $H_{1}: C_{L}>c^{*}$, is constructed. 
The value of test statistic is $\hat{C}_{L}=0.888$, the critical value $C_{0}^{*}=0.873$ by using (21). Since $\hat{C}_{L}=0.888>C_{0}^{*}=0.873$, so we reject the null hypothesis $H_{0}: C_{L} \leq c^{*}$. Also, the lower confidence bound is $(0.824, \infty)$. So, $c^{*}=0.80$ don't belong to lower bound, it is also concluded that the lifetime performance index of airplane components meets the required level.

\section{Conclusions Remarks}

The lifetime performance index $C_{L}$ was utilized to evaluate the performance of a process. In this paper, we consider the estimation problem of $C_{L}$ on the basis of progressively Type II censored samples. Based on the Burr III distribution for lifetimes of products, the ML estimate of $C$ is obtained. These estimators of $C_{L}$ is used to construct $100(1-\gamma) \%$ lower confidence bounds as well as a test of the null hypothesis $H_{0}: C_{L} \leq c^{*}$, versus $H_{1}: C_{L}>c^{*}$, based on maximum likelihood procedure. These hypothesis procedures may be used for assessing whether the product performance meets customer expectations or not. Simulation study is performed and a real data set of airplane components is analyzed. Based on a numerical study, we notice that the MSEs of the $\hat{C}_{L}$ estimates decrease as the sample size increases for selected samples schemes. Also, the performance of our proposed method is acceptable.

\section{References}

1. Ahmadi,V. M., Doostparast, M. and Ahmdi, J. (2013). Estimating the lifetime performance index with Weibull distribution based on progressive first-failure censoring scheme. Journal of Computational and Applied Mathematics, 239, 93-102.

2. Altindag, O., Ankaya, M. N., Yalinkaya, A. and Aydogdu, H. (2017). Statistical inference for the Burr Type III distribution under Type II censored data. Communication Science. 66(2), 297-310.

3. Burr, W. I. (1942).Cumulative frequency functions. The Annals of Mathematical Statistics, 13(2), 215-232.

4. Cohen, A. C. (1965). Maximum likelihood estimation in the Weibull distribution based on complete and cesored samples.Technometrics, 7( 4), 579-588.

5. Gamchi, F. V., Alma, O. G. and Belaghi, R. A. (2019). Classical and Bayesian inference for Burr Type-III distribution based on progressive Type-II hybrid censored data. Mathematical Science, 13(2),79-95.

6. Gildeh, S. B. and Nadi, A. A. (2016). Estimating the lifetime performance index of products for twoparameter exponential distribution with the progressive first-failure censored sample, International Journal for Quality Research, 10(2), 389-406.

7. Hassanein, A. W. (2018). The lifetime performance index of power lomax distribution based on progressive first-failure censoring scheme. Journal of Statistics Applications \& Probability An International Journal, 7(2), 333-341.

8. Hong, C. W., Lee, C. W. and Wu, J. W. (2012). Computational procedure of performance assessment of lifetime index of products for the weibull distribution with the progressive first-failure-censored sampling plan. Journal of Applied Mathematics, Article ID 717184, 13 pages.

9. Kane, V. E., (1986).Process capability indices. Journal of Quality Technology, 18, 41-52.

10. Lee, C. W., Wu, W. J. and Hong, W. C. (2009). Assessing the lifetime performance index of products with the exponential distribution under progressively type II right censored samples. Journal of Computational and Applied Mathematics, 231, 648-656.

11. Mann, N. R. and Fertig, K. W. (1973). Tables for obtaining Weibull condence bounds and tolerance bounds based on best linear invariant estimates of parameters of the extreme-value distribution. Technometrics 15(1), 87-101.

12. Montgomery, D. C. (1985). Introduction to Statistical Quality Control. John Wiley and Sons Inc., New York.

13. Panahi, H. (2017). Estimation of the Burr Type III distribution with application in unified hybrid censored sample of fracture toughness. Journal of Applied Statistics, 44, 2575-2592.

14. Viveros, R. and Balakrishnan, N. (1994). Interval estimation of parameters of life from progressively censored data, Technometrics, 36, 84-91. 
15. Wijekularathna, D. K. and Subedi, N. (2019). Implementing the lifetime performance index of products with a two-parameter Rayleigh distribution under a progressively Type II right censored sample. Journal of Mathematics and Statistics, 5, 28-40.

16. Wu, C. C., Chen, L. C. and Chen, Y. J. (2013). Decision procedure of lifetime performance assessment of Rayleigh products under progressively Type II right censored samples. International Journal of Information \& Management Sciences, 24(3), 225-237.

17. Wu, J. W., Lee, W. C., Hong, C. W. and Yeh, S. Y. (2014). Implementing lifetime performance index of Burr XII products with progressively type II right censored sample. International Journal of Innovative Computing, Information and Control, 10(2), 671-693. 